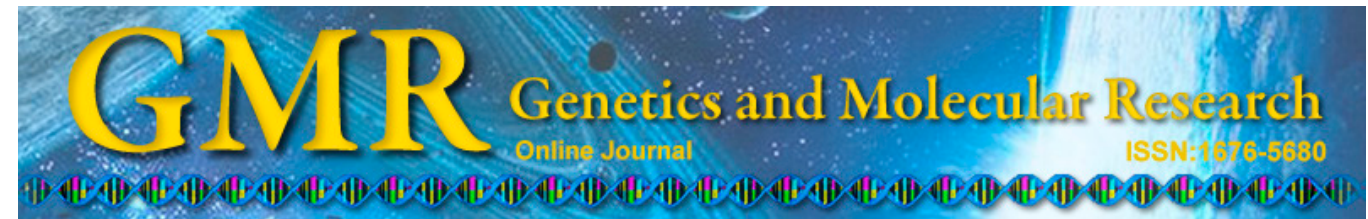

\title{
MTHFR C677T and A1298C polymorphisms as predictors of radiotherapy response in head and neck squamous cell carcinoma
}

\author{
Q.S. Anders ${ }^{1}$, E. Stur ${ }^{1}$, L.P. Agostini ${ }^{1}$, F.M. Garcia ${ }^{1}$, R.S. Reis ${ }^{1}$, J.A. Santos ${ }^{1}$, \\ S.O. Mendes ${ }^{1}$, L.L. Maia ${ }^{5}$, G.T. Peterle ${ }^{5}$, V. Stange ${ }^{1}$, M.B. Carvalho ${ }^{2}$, \\ E.H. Tajara ${ }^{3}$, M. Santos ${ }^{4}$, A.M.A. Silva-Conforti ${ }^{1,5}$ and I.D. Louro ${ }^{1}$ \\ ${ }^{1}$ Núcleo de Genética Humana e Molecular, \\ Departamento de Biologia, Universidade Federal do Espírito Santo, \\ Espírito Santo, ES, Brasil \\ ${ }^{2}$ Laboratório de Biologia Molecular, Hospital Heliópolis, \\ São Paulo, SP, Brasil \\ ${ }^{3}$ Departamento de Biologia Molecular, Faculdade de Medicina, \\ São José do Rio Preto, SP, Brasil \\ ${ }^{4}$ Departamento de Medicina, Universidade Federal do Rio Grande do Norte, \\ Campus Caicó, Caicó, RN, Brasil \\ ${ }^{5}$ Departamento de Biologia, Universidade Federal do Espírito Santo, \\ Alegre, ES, Brasil \\ Corresponding author: I.D. Louro \\ E-mail: iurilouro@yahoo.com
}

Genet. Mol. Res. 14 (4): 13105-13109 (2015)

Received June 5, 2015

Accepted September 17, 2015

Published October 26, 2015

DOI http://dx.doi.org/10.4238/2015.October.26.6

\begin{abstract}
The C677T and A1298C polymorphisms in methylenetetrahydrofolate reductase (MTHFR), which regulates the release of active folate in the body, may have reduced activity. Given that folate participates in important intracellular pathways, such as nucleotide synthesis and biomolecule methylation, it seems plausible that patients with head and neck squamous cell carcinoma (HNSCC) may respond differently to radiotherapy treatments, based on genetic polymor-
\end{abstract}


phisms. Therefore, this study sought to understand the role of these polymorphisms in HNSCC patient radiotherapy response. Genotypes were detected by PCR-RFLP after extraction of DNA from peripheral blood lymphocytes. Survival curves were analyzed by the KaplanMeier model, and significant differences were analyzed by the Wilcoxon test. Response to radiotherapy in patients with laryngeal SCC was significantly associated with the MTHFR C677T polymorphism $(\mathrm{P}=0.030)$. Indeed, the presence of at least one $\mathrm{T}$ allele decreases the mortality rate up to 3 -fold. Therefore, we propose that MTHFR C677T may represent a putative biomarker for radiotherapy prognosis in laryngeal SCC patients.

Key words: MTHFR; Head and neck cancer; Radiotherapy

\section{INTRODUCTION}

Head and neck squamous cell carcinoma (HNSCC) describes malignant tumors of the upper aerodigestive tract. This region includes the oral cavity, pharynx, larynx, maxillary sinus, nasal cavity, and tumors of the salivary glands and thyroid (Dobrossy, 2005).

Micronutrient deficiency may be associated with increased risk of HNSCC, whereas vegetal rich diets probably decrease this risk. This is likely due to components present in vegetables that induce transcription of antioxidant enzymes (Gigliotti et al., 2008).

Folate is an essential nutrient for purine synthesis, and its deficiency interferes with adequate DNA and RNA synthesis. Such deficiency is normally caused by acetaldehyde, an ethanol metabolite, and by tobacco components (Vannucchi and Melo, 2010). Due to its role in nucleotide synthesis, the enzyme that converts folate into its active form, methylenetetrahydrofolate reductase (MTHFR), may have a role in carcinogenesis, as variants with different activities can alter purine metabolism and patient prognosis (Nazki et al., 2014). The MTHFR polymorphisms C677T and A1298C have reduced enzymatic activity, altering folate bioavailability and purine metabolism (Baluz et al., 2002; Galbiatti et al., 2012).

Radiotherapy treatment in cancer causes tumor regression and increases disease-free survival, as compared to surgery alone (Moore et al., 2013). This study sought to understand the influence of MTHFR polymorphisms on radiotherapy response in HNSCC patients, by analyzing MTHFR C677T (rs1801133) and A1298C (rs1801131).

\section{MATERIAL AND METHODS}

\section{Subjects}

We analyzed 306 patients with oral, oropharyngeal, and laryngeal squamous cell carcinoma treated at the Head and Neck Surgery Service at Hospital Heliópolis from 2001 to 2011. Among these, 157 patients underwent operation and irradiation, whereas 149 underwent operation only. Our results were organized according to tumor type, with 206 oral and oropharyngeal SCC and 100 laryngeal SCC.

Among all patients, 40 were females, and 266 were males. The mean age was 57 
years old. For the prognosis analysis, all patients were classified according to tumor stage, pathological classification, lymph node presence, and local and general disease relapse and death. In regards to radiotherapy treatment, 157 patients were treated with radiotherapy, of which 101 were patients with oral or oropharyngeal SCC, whereas 48 were laryngeal tumor patients.

Inclusion criteria included were a follow-up of at least 24 months, being metastasis free, and only surgical treatment prior to sample collection.

This study was approved by the Ethics Committee of the Hospital Heliópolis (São Paulo/Brazil), under protocol number 135.

\section{Genotyping}

Peripheral blood DNA was extracted using the salting out method. The target region was amplified by PCR, digested, separated by $3 \%$ agarose gel electrophoresis, and stained with ethidium bromide. Table 1 shows the primer sequence, fragment size, enzymes used, and cycling conditions.

Table 1. PCR-RFLP conditions for the two polymorphisms.
\begin{tabular}{llllc}
\hline Polymorphism & Primer sequence & Fragment (bp) & Enzyme & Annealing temp/No. of cycles \\
\hline MTHFR & 5'-AGGACGGTGCGGTGAGAGTG-3' & 198 & Hinfl & $60^{\circ} \mathrm{C} / 60^{\prime \prime}-40 \mathrm{X}$ \\
677C $>$ T (Ala222Val) & 5'-TGAAGGAGAAGGTGTCTGCGGA-3' & & & \\
MTHFR & 5'-CTTTGGGGAGCTGAAGGACTAC-3' & 163 & MboII & $60^{\circ} \mathrm{C} / 30^{\prime \prime}-40 \mathrm{X}$ \\
1298A $>$ C (Glu430Ala) & 5'-ACTTTGTGACCATTCCGGTTTG-3' & & & \\
\hline
\end{tabular}

The Lilliefors significance test was used $(\mathrm{P}<0.05)$. The chi-square test and, when necessary, the Fisher exact test were used for association studies. Survival curves were evaluated according to the Kaplan-Meier model, and significance was confirmed using the Wilcoxon test.

Multivariate logistic regression and Cox proportional Hazard were used to adjust the $\mathrm{OR}$, hazard ratio, and $95 \% \mathrm{CI}$ for significant results. Clinico-biological relations and $\mathrm{P}$ values less than 0.10 were considered to be significant in the multivariate analyses. Statistical calculations were performed using the Epi-Info ${ }^{\circledR}$ v3.4.3 software.

\section{RESULTS}

Analysis of MTHFR C677T and A1298C in HNSCC patients treated with radiotherapy showed no association with local disease relapse, death, or local disease-free survival. We further evaluated specific disease survival. Interestingly, in laryngeal SCC patients treated with radiotherapy, we observed a significant relationship between survival and MTHFR C677T (P $=0.030$; Figure 1), which was not observed in non-irradiated patients (data not shown). As shown in Figure 1, after 12 months, approximately $27 \%$ of patients with the $\mathrm{C}$ allele had died, whereas $8 \%$ of $\mathrm{T}$ allele patients had died. Multivariate analysis confirmed these results, and showed that the presence of at least one $\mathrm{T}$ allele reduces the risk of early death 3 -fold (hazard ratio $=0.30 ; 95 \% \mathrm{CI}=0.10-0.89$; Table 2 ). 


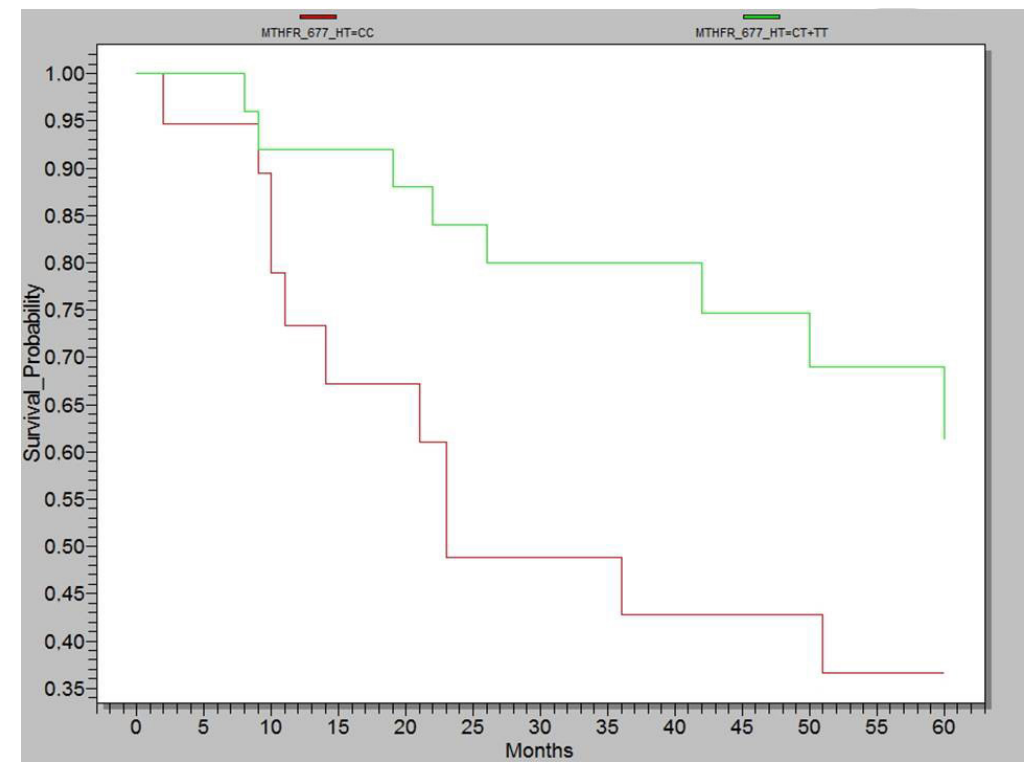

Figure 1. Disease-free survival curve in laryngeal SCC patients treated with radiotherapy, according to MTHFR C677T variants $(\mathrm{N}=19$ patients, $\mathrm{P}=0.030)$.

Table 2. Multivariate analysis of disease-specific survival of irradiated laryngeal SCC patients, according to MTHFR C677T polymorphism genotypes.

\begin{tabular}{lcc}
\hline Variables & HR $(95 \% \mathrm{CI})$ & P \\
\hline Stage & 1 & 0.685 \\
I, II & $1.72(0.13-23.69)$ & 0.960 \\
III & $0.95(0.12-7.63)$ & \\
IV & 1 & 0.030 \\
MTHFR C677T (Ala/Val) & $0.30(0.10-0.89)$ & \\
CC (Ala/Ala) & 1 & 0.407 \\
CT + TT (Ala/Val+Val/Val) & $0.66(0.25-1.75)$ & \\
MTHFR A1298C (Glu/Ala) & & \\
AA (Glu/Glu) & & \\
AC + CC (Glu/Ala+Ala/Ala) &
\end{tabular}

\section{DISCUSSION}

This study evaluated the hypothesis that MTHFR C677T and A1298C polymorphisms were associated with HNSCC prognosis after radiotherapy. Most prognostic characteristics had no association with tumor location. However, laryngeal SCC patients treated with chemotherapy showed an association between MTHFR C677T and survival, which was confirmed by multivariate analysis. Our study demonstrated that the presence of at least one $\mathrm{T}$ allele decreased mortality 3 -fold.

Sailasree et al. (2011) evaluated 130 patients with oral SCC, and found that the A1298C polymorphism was associated with lower survival and increased disease relapse. However, we did not find this in our study. In the same study, the C677T polymorphism was not associated with survival. Nonetheless, a trend towards increased survival was observed, which aligned with our data. The difference in significance may be due to sample specificity, 
since the previous study evaluated patients treated with radiotherapy and chemotherapy, with no treatment modality separation. This may be of crucial importance, as 5FU inhibits DNA synthesis, similar to low concentrations of folate.

Considering the protective effect of the MTHFR 677T allele in irradiated laryngeal SCC, we hypothesize that inefficient MTHFR activity caused by the amino acid substitution (Ala/Val) increases tumor radiosensitivity, rendering the treatment more efficient.

These results indicate that MTHFR deficiency caused by polymorphisms decrease folate bioavailability in the tumor microenvironment, affecting nucleotide synthesis, proliferation, and repair.

Kawakita et al. (2012) analyzed 277 patients with HNSCC, but did not find any association between the $\mathrm{C} 677 \mathrm{~T}$ polymorphism and prognosis. However, their sample population was composed of patients treated with radiotherapy and chemotherapy, and was therefore substantially different from ours.

\section{CONCLUSION}

Based on these results, we propose that the MTHFR C677T polymorphism is a prognostic biomarker for radiotherapy treatment in laryngeal SCC, as the presence of at least one $\mathrm{T}$ allele in the MTHFR gene increases disease-specific survival.

\section{Conflicts of interest}

The authors declare no conflict of interest.

\section{ACKNOWLEDGMENTS}

Research supported by Conselho Nacional de Desenvolvimento Científico e Tecnológico (CNPq), Fundação de Amparo à Pesquisa do Estado do Espírito Santo (FAPES), and Coordenação de Aperfeiçoamento de Pessoal de Nível Superior (CAPES).

\section{REFERENCES}

Baluz K, Carmo MGT and Rosas G (2002). O papel do ácido fólico na prevenção e na terapêutica oncológica: revisão. Rev. Bras. Cancerol. 48: 597-607.

Dobrossy L (2005). Epidemiology of head and neck cancer: Magnitude of the problem. Cancer Met. Rev. 24: 9-17.

Galbiatti ALS, Ruiz MT, Maniglia JV, Raposo LS, et al. (2012). Head and neck cancer: genetic polymorphisms and folate metabolism. Braz. J. Otorhinolaryngol. 78: 132-139.

Gigliotti MP, Tolentino ES, Tomita NE and Chinellato LEM (2008). Principais mecanismos de atuação do álcool no desenvolvimento do câncer oral. Odontol. Clín.-Cientif. 7: 107-112.

Kawakita D, Matsuo K, Sato F, Oze I, et al. (2012). Association between dietary folate intake and clinical outcome in head and neck squamous cell carcinoma. Ann. Oncol. 23: 186-192.

Moore K, Ford P and Farah C (2013). Support needs and quality of life in oral cancer: a systematic review. Int. J. Dent. Hyg. 12: 36-47.

Nazki FH, Sameer AS and Ganaie BA (2014). Folate: Metabolism, genes, polymorphisms and the associated diseases. Gene 533: 11-20.

Sailasree R, Nalinakumari KR, Sebastian P and Kannan S (2011). Influence of methylenetetrahydrofolate reductase polymorphisms in oral cancer patients. J. Oral Pathol. Med. 40: 61-66.

Vannucchi H and Melo SS (2009). Hiper-homocisteinemia e risco cardiometabólico. Arq. Bras. Endocrinol. Metab. 53: 540-549. 